\title{
Quantum Remote Control: Teleportation of Unitary Operations
}

\author{
S.F. Huelga*, J.A. Vaccaro and A. Chefles \\ Department of Physical Sciences, University of Hertfordshire, Hatfield AL10 9AB, England \\ M.B. Plenio \\ Optics Section, The Blackett Laboratory, Imperial College, London SW7 2BW, England
}

(February 1, 2008)

\begin{abstract}
We consider the implementation of an arbitrary unitary operation $U$ upon a distant quantum system. This teleportation of $U$ can be viewed as a quantum remote control. We investigate protocols which achieve this using local operations, classical communication and shared entanglement (LOCCSE). Lower bounds on the necessary entanglement and classical communication are determined using causality and the linearity of quantum mechanics. We examine in particular detail the resources required if the remote control is to be implemented as a classical black box. Under these circumstances, we prove that the required resources are, necessarily, those needed for implementation by bidirectional state teleportation.
\end{abstract}

PACS-numbers: 03.67.-a, 03.65.Bz 
Much of the current fascination with quantum information processing derives from the properties of entanglement [1]. On one hand, entanglement can give rise to nonlocal correlations which defy explanation in terms of local, realistic theories [2], but on the other, it can also be used as a resource. While it is impossible, for example, to determine the state of a quantum system, entanglement makes it possible to transmit an unknown state. This process is known as quantum state teleportation [3]. Quantum state teleportation can be linked directly to various interrelated principles of quantum information processing, such as the impossibility of superluminal communication, the non-increasing of entanglement under local operations and classical communication [1] and the no-cloning theorem [4]. However, the information contained in the state of a quantum system is only one kind of information which is important in quantum mechanics. Another is the information which describes quantum operations. In this paper, we examine the issue of teleporting, not a quantum state, but rather a quantum operation. In particular, we examine the teleportation of an unknown unitary operation on a qubit. This procedure would function in a manner similar to that of a remote control apparatus, and so we shall also refer to it as quantum remote control. We will first pose the problem in a completely general theoretical framework and focus later on an experimentally feasible scenario where entanglement resources are limited. The most general scenario for the teleportation of an arbitrary unitary operation is depicted in Figure 1. One party, Alice, possesses a physical system, $C$, which we shall refer to as the control. The control contains information describing a unitary operation $U$ upon the state of a qubit, and is itself a quantum system. The control state corresponding to the unitary operation $U$ will be denoted by $|U\rangle_{C}$. Her colleague Bob has a qubit $\beta$ prepared in the state $|\psi\rangle_{\beta}$. The aim is to devise a physical procedure which effects the transformation $|\psi\rangle_{\beta} \mapsto U|\psi\rangle_{\beta}$, for every initial state $|\psi\rangle_{\beta}$ and every unitary operation $U$. The most general such procedure can be represented by a completely positive, linear, trace preserving map on the set of density operators for the combined $C \beta$ system. Any such map has a unitary representation $\mathcal{T}$ involving ancillary systems. We shall denote the state of the ancilla at Alice's and Bob's laboratories by $|\chi\rangle_{A B}$. Then the teleportation operation has the general form

$$
\mathcal{T}\left[|\chi\rangle_{A B} \otimes|U\rangle_{C} \otimes|\psi\rangle_{\beta}\right]=|\Phi(U, \chi)\rangle_{A B C} \otimes\left(U|\psi\rangle_{\beta}\right) .
$$

In the following we investigate some of the properties of $\mathcal{T}$. In particular, we derive lower bounds on the amount of non-local resources that are needed to implement $\mathcal{T}$ using only local operations and classical communication. The unitary teleportation operator $\mathcal{T}$ is independent of both $U$ and $|\psi\rangle_{\beta}$. The final state of the ancilla+control, $|\Phi(U, \chi)\rangle_{A B C}$, must be independent of $|\psi\rangle_{\beta}$. To see why, let us suppose that it isn't, in which case there will be at least one $U$, and two states, $|\psi\rangle_{\beta}$ and $\left|\psi^{\prime}\right\rangle_{\beta}$, for which $|\Phi(U, \chi, \psi)\rangle_{A B C} \neq\left|\Phi\left(U, \chi, \psi^{\prime}\right)\right\rangle_{A B C}$. We imagine that $U$ is successfully teleported for the states $|\psi\rangle_{\beta}$ and $\left|\psi^{\prime}\right\rangle_{\beta}$. Suppose now that Bob's qubit is prepared in a superposition of these states, $\left(c_{1}|\psi\rangle+c_{2}\left|\psi^{\prime}\right\rangle\right)_{\beta}$. The linearity of $\mathcal{T}$ implies that the final total state will be

$$
\left(\mathbb{1}_{A B C} \otimes U_{\beta}\right)\left[c_{1}|\Phi(U, \chi, \psi)\rangle_{A B C} \otimes|\psi\rangle_{\beta}+c_{2}\left|\Phi\left(U, \chi, \psi^{\prime}\right)\right\rangle_{A B C} \otimes\left|\psi^{\prime}\right\rangle_{\beta}\right] .
$$

The requirement that Bob's qubit undergoes a unitary evolution implies that it must not be entangled with the remaining systems. However, one can see that it is entangled with ABC 
whenever $c_{1} c_{2} \neq 0$. Thus, the final state of $\mathrm{ABC}$ must be independent of $|\psi\rangle_{\beta}$.

The set of all unitary operations $U$ is infinite. This implies that if the dimension of the control system is to be finite, then the control states $|U\rangle_{C}$ must, in general, be non-orthogonal. However, Nielsen and Chuang showed, in a slightly different context, that this cannot be the case [5]. The problem investigated by these authors was whether or not one could devise a universal programmable quantum gate array, which could be used to store and execute any program upon a quantum register. They showed that no such finite array can be constructed. Their method of proof can readily be transferred to this context, making use of the correspondences between programmable gate array/control, and register/Bob's qubit. Following their reasoning, we note that Eq. (1) and the unitarity of $\mathcal{T}$ imply that, for any two different unitary transformations $U$ and $U^{\prime}$,

$$
\frac{C\left\langle U^{\prime} \mid U\right\rangle_{C}}{{ }_{A B C}\left\langle\Phi\left(U^{\prime}, \chi\right) \mid \Phi(U, \chi)\right\rangle_{A B C}}={ }_{\beta}\left\langle\psi\left|U^{\prime \dagger} U\right| \psi\right\rangle_{\beta} .
$$

The left hand side is independent of $|\psi\rangle_{\beta}$, and this equality is true for all $|\psi\rangle_{\beta}$. It follows that $U^{\prime} \dagger=\gamma \mathbb{1}$, for some constant $\gamma$, leading to the conclusion that $U$ and $U^{\prime}$ are identical up to a multiplicative constant. This conclusion, however, is valid only when the denominator on the left hand side is non-zero. If it is zero, then ${ }_{C}\left\langle U^{\prime} \mid U\right\rangle_{C}=0$, by the unitarity of $\mathcal{T}$. Control states corresponding to different unitary transformations are orthogonal, so that no finite-dimensional control system can be used to teleport an arbitrary unitary operation. For the remainder of this paper, when we speak of an arbitrary unitary operation, we will mean one which belongs to some arbitrarily large, but finite, set. We will also assume that this set contains the identity $\sigma^{0}=\mathbb{1}$ and the 3 Pauli operators $\sigma^{i}$. Note that the orthogonality of the control states opens the possibility that different operations can, at least in principle, be distinguished by Alice. This will only be possible though if Alice knows the basis $\left\{|U\rangle_{C}\right\}$ in which the information is encoded.

The teleportation of $U$ is a collective operation on spatially separated systems, which we wish to carry out using shared entanglement and classical communication. In the derivation of lower bounds on the amount of non-local resources that are required to implement the teleportation of $U$ locally, two guiding principles will be very useful [1]:

(i) The amount of classical information able to be communicated by an operation in a given direction across some partition between subsystems cannot exceed the amount of information that must be sent in this direction across the same partition to complete the operation.

(ii) The amount of bipartite entanglement that an operation can establish across some partition between subsystems cannot exceed the amount of prior entanglement across the partition that must be consumed in order to complete the operation.

We now use principle (i) to establish the fact that at least two classical bits must be sent from Alice to Bob to complete the teleportation of an arbitrary $U$. Suppose that, rather than being prepared in a pure state, Bob's qubit is initially maximally entangled with some other qubit, $\beta^{\prime}$, which is also in Bob's laboratory. Let us denote the four Bell states for a pair of qubits by $\left|B^{\mu}\right\rangle$, where $\mu=0, \ldots, 3$. Using the technique of super-dense coding [6], any of the four Bell states can be transformed into any other by application of one of the Pauli operators $\sigma^{i}$ on one of the qubits. We take this qubit to be $\beta$, and notice that the $\left|B^{\mu}\right\rangle$ can be ordered in such a way that $\left(\sigma_{\beta}^{\mu} \otimes \mathbb{1}_{\beta^{\prime}}\right)\left|B^{0}\right\rangle_{\beta \beta^{\prime}}=\left|B^{\mu}\right\rangle_{\beta \beta^{\prime}}$. Alice can easily transmit two 
bits of information to Bob if he prepares the $\beta \beta^{\prime}$ system in the state $\left|B^{0}\right\rangle_{\beta \beta^{\prime}}$. She chooses the control system to be in one of the states $\left|\sigma^{\mu}\right\rangle_{C}$. Following the action of $\mathcal{T}$, Bob will be in possession of the corresponding Bell state $\left|B^{\mu}\right\rangle_{\beta \beta^{\prime}}$. If he subsequently performs a Bell measurement on $\beta \beta^{\prime}$, then he will be able to determine the value of $\mu$, and hence the control state which Alice prepared, revealing 2 bits of classical information.

We now show that, by teleporting an arbitrary $U$ according to the general prescription in Eq. (1), Alice and Bob can establish 2 ebits of shared entanglement. Imagine that, in addition to the systems we have already introduced, Alice has a further 4-dimensional ancilla, which we shall label $R$. Let the states $|\mu\rangle_{R}$ be a particular orthonormal basis for $R$. Suppose now that Alice initially prepares $R$ and the control $C$ in the maximally entangled state $(1 / 2) \sum_{\mu}|\mu\rangle_{R} \otimes\left|\sigma^{\mu}\right\rangle_{C}$. Bob once more prepares the Bell state $\left|B^{0}\right\rangle_{\beta \beta^{\prime}}$. The teleportation operation $\mathcal{T}$ is then carried out according to Eq. (1). It is more convenient here, however, to work with a form of this equation that represents, explicitly, any local measurements made by Alice and Bob and any classical communication between them. In this case $\mathcal{T}$ in Eq. (1) is replaced by a pair of classically-correlated local CP maps, one in each laboratory. Classical information is revealed by measurements, and we let the index $i$ denote each measurement outcome. The final state corresponding to the ith outcome is

$$
\left|\psi_{F}\right\rangle_{i}=\frac{1}{2} \sum_{\mu}|\mu\rangle_{R} \otimes\left|\Phi_{i}\left(\sigma^{\mu}, \chi\right)\right\rangle_{A B C} \otimes\left|B^{\mu}\right\rangle_{\beta \beta^{\prime}}
$$

We now calculate the entanglement shared by Alice and Bob. Alice is in possession of the compound system $R A C$, while Bob has the system $B \beta \beta^{\prime}$. For each outcome, these subsystems have respective density operators $\rho_{R A C}^{i}$ and $\rho_{B \beta \beta^{\prime}}^{i}$. Since $\left|\psi_{F}\right\rangle_{i}$ is a pure state, it follows that the entanglement shared by Alice and Bob is simply the (base 2) von Neumann entropy of either of these density operators. Fortunately, we can calculate this explicitly. To do so, we notice that the states $\left|\Phi_{i}\left(\sigma^{\mu}, \chi\right)\right\rangle_{A B C}$ will generally contain entanglement between $B$ and $A C$. Let us write $\rho_{B}^{i \mu}=\operatorname{Tr}_{A C}\left(\left|\Phi_{i}\left(\sigma^{\mu}, \chi\right)\right\rangle\left\langle\Phi_{i}\left(\sigma^{\mu}, \chi\right)\right|\right)$. We find that [7]

$$
\rho_{B \beta \beta^{\prime}}=\frac{1}{4} \sum_{\mu}\left(\left|B^{\mu}\right\rangle\left\langle B^{\mu}\right|\right)_{\beta \beta^{\prime}} \otimes \rho_{B}^{i \mu} .
$$

Making use of the orthogonality of the $\left|B^{\mu}\right\rangle$, we find that the total entropy of entanglement shared by Alice and Bob is simply

$$
E\left(\left|\psi_{F}\right\rangle\right)=S\left(\rho_{B \beta \beta^{\prime}}\right)=2+\frac{1}{4} \sum_{\mu} S\left(\rho_{B}^{i \mu}\right) \geq 2 .
$$

It follows from principle (ii) that at least 2 ebits of entanglement need to be consumed to implement $\mathcal{T}$ locally, i.e. to teleport an arbitrary unitary operation.

We can summarize the results obtained so far as follows. The resources required to perform quantum remote control can be classified into shared entanglement, classical information transmission from Alice to Bob, and from Bob to Alice. We have established absolute lower bounds on the first two of these resources. Alice and Bob have to share at least two ebits and Alice needs to transmit to Bob, at least, two bits of classical information.

These bounds can be attained by a procedure in which Bob teleports the state of his particle to Alice who, after applying the unitary transformation, teleports it back to him. 
We will call this the "bidirectional state teleportation" scheme. The scheme requires sending 2 classical bits in each direction, and using 2 ebits of shared entanglement. It would also be conceivable to adopt a different strategy - teleporting the state of the control system from Alice to Bob who would then implement the control directly onto $\beta$. We call this the "control-state teleportation" scheme.

Control-state teleportation is a unidirectional communication scheme from Alice to Bob, so the absolute lower bound for the communication exchange from Bob to Alice is zero. Obviously, the overall resources will depend on the dimensionality of the control system $C$. We cannot say anything about the optimality of this procedure; whether there exists another unidirectional protocol which uses less resources is an open problem.

On the other hand, bidirectional state teleportation saturates the lower bounds for the amount of shared ebits and classical bits transmitted from Alice to Bob and additionally uses two bits of classical communication from Bob to Alice. This scheme allows the faithful implementation of $U$ independently of the dimension of the control system. To be more efficient overall, any other scheme would need less resources than bidirectional state teleportation. This establishes an upper bound in the overall amount of resources required for the efficient remote implementation of an arbitrary $U$ as 4 classical bits and 2 ebits.

We now consider an experimental scenario where the black box implementing an arbitrary transformation $U$ is a macroscopic object, involving a (very) large number of degrees of freedom. The option of teleporting the control apparatus is then unfeasible, given that it would consume an infinite amount of entanglement and classical communication resources. However, the question remains whether there exists a more economical protocol than bidirectional state teleportation. We will prove in the following that this is not possible and bidirectional state teleportation is an unconditional optimal way to remotely implement an arbitrary $U$.

Discarding the possibility of control-state teleportation allows us to replace the transformation given by Eq. (II) with

$$
G_{2} U G_{1}\left(|\chi\rangle_{\alpha A B} \otimes|\psi\rangle_{\beta}\right)=|\Phi(U, \chi)\rangle_{\alpha A B} \otimes U|\psi\rangle_{\beta},
$$

where certain fixed operations $G_{1}$ and $G_{2}$ are performed, respectively, prior to and following the action of the arbitrary $U$ on a qubit $\alpha$ on Alice's side. We assume that Alice and Bob share initially some entanglement, represented by the state $|\chi\rangle_{\alpha A B}$. As before, the purpose of the transformation is to perform the operation $U$ on Bob's qubit $\beta$. We continue to use a nonlocal unitary representation of the transformation where $G_{1}$ and $G_{2}$ are unitary operators acting on possibly all subsystems. A pictorial scheme of the situation using a quantum circuit is given in Figure 2. The two upper wires refer to Alice's subsystems and the two lower ones to Bob's. Note that operations $G_{i}$ are represented by non-local gates while the action of $U$ takes place locally on Alice's side.

We prove in the following that the only way that Eq. (7) can be implemented (locally) is by teleporting the state $|\psi\rangle_{\beta}$ from Bob to Alice, and then teleporting back the transformed state $U|\psi\rangle_{\beta}$ from Alice to Bob.

We begin by noting that linearity forces the transformed state of systems $\alpha A B$ to be independent of the particular input state $|\psi\rangle_{\beta}$. In addition, linearity imposes the condition that the state $|\Phi(U, \chi)\rangle_{\alpha A B}$ has to be independent of $U$ itself. To see this, consider the case 
where the transformation $U$ is one of the four Pauli operators $\sigma^{\mu}$ and assume that the global state of $\alpha A B$ after completing the protocol may depend on the choice of $U$. According to Eq. (7), the combined action of the operations $G_{i}$ has to be such that

$$
G_{2} \sigma^{\mu} G_{1}\left(|\chi\rangle_{\alpha A B} \otimes|\psi\rangle_{\beta}\right)=\left|\Phi\left(\sigma^{\mu}, \chi\right)\right\rangle_{\alpha A B} \otimes\left(\sigma^{\mu}|\psi\rangle_{\beta}\right) .
$$

On the other hand, an arbitrary one-qubit unitary transformation $U$ can always be decomposed in terms of the Pauli operators, $U=\sum_{\mu=0}^{4} \alpha_{\mu} \sigma^{\mu}$, and it must hold that

$$
G_{2} U G_{1}\left(|\chi\rangle_{\alpha A B} \otimes|\psi\rangle_{\beta}\right)=\sum_{\mu} \alpha_{\mu}\left|\Phi\left(\sigma^{\mu}, \chi\right)\right\rangle_{\alpha A B} \otimes\left(\sigma^{\mu}|\psi\rangle_{\beta}\right) .
$$

For the RHS to be a product state, as is required by Eq. (7), we must have $\left|\Phi\left(\sigma^{\mu}, \chi\right)\right\rangle_{\alpha A B}=$ $|\Phi(\chi)\rangle_{\alpha A B}$, independent of the operator $\sigma^{\mu}$. This is true for any basis set of operators, and so the final state of the ancillas $\alpha A B$ on the RHS of Eq. (7) is independent of $U$.

We can now show that the operation $G_{1}$ necessarily has to be non-trivial. We do this by first assuming the contrary that $G_{1}=\mathbb{1}$, and considering two input states, $|\psi\rangle_{\beta}$ and $\left|\psi^{\prime}\right\rangle_{\beta}$ such that $\beta_{\beta}\left\langle\psi^{\prime} \mid \psi\right\rangle_{\beta}=0$, and two unitary transformations $U$ and $U^{\prime}$ which bring these two states to the same state $|\gamma\rangle_{\beta}$. Using Eq. (1), this implies that

$$
\begin{aligned}
G_{2}\left(U|\chi\rangle_{\alpha A B}|\psi\rangle_{\beta}\right) & =|\Phi(\chi)\rangle_{\alpha A B} \otimes|\gamma\rangle_{\beta} \\
G_{2}\left(U^{\prime}|\chi\rangle_{\alpha A B}\left|\psi^{\prime}\right\rangle_{\beta}\right) & =|\Phi(\chi)\rangle_{\alpha A B} \otimes|\gamma\rangle_{\beta} .
\end{aligned}
$$

No universal unitary action $G_{2}$ can be found to satisfy Eq. (10), as this would require the mapping of orthogonal states onto the same state. This shows that no universal operation $G_{2}$ that satisfies Eq. (10) can exist and therefore, for the $U$-teleportation to succeed, $G_{1}$ has to be non-trivial.

The final step in our proof is to rewrite Eq. (17) as

$$
U G_{1}\left(|\chi\rangle_{\alpha A B} \otimes|\psi\rangle_{\beta}\right)=G_{2}^{\dagger}\left(|\Phi(\chi)\rangle_{\alpha A B} \otimes U|\psi\rangle_{\beta}\right)
$$

Since $G_{1}$ and $G_{2}$ are universal gates, we may choose $U$ and $|\psi\rangle_{\beta}$ freely. For each $|\psi\rangle_{\beta}$ let the operator $U_{\psi}$ be such that $U_{\psi}|\psi\rangle=|0\rangle$ where $\sigma_{z}|0\rangle=|0\rangle$. If $U=\sigma_{z} U_{\psi}$, then

$$
\begin{aligned}
\left(\sigma_{z} U_{\psi}\right) G_{1}\left(|\chi\rangle_{\alpha A B} \otimes|\psi\rangle_{\beta}\right) & =G_{2}^{\dagger}\left(|\Phi(\chi)\rangle_{\alpha A B} \otimes \sigma_{z} U_{\psi}|\psi\rangle_{\beta}\right) \\
& =G_{2}^{\dagger}\left(|\Phi(\chi)\rangle_{\alpha A B} \otimes|0\rangle_{\beta}\right) .
\end{aligned}
$$

The RHS is simply $\left(U_{\psi}\right) G_{1}\left(|\chi\rangle_{\alpha A B} \otimes|\psi\rangle_{\beta}\right)$ and so, necessarily, $\left(U_{\psi}\right) G_{1}\left(|\chi\rangle_{\alpha A B} \otimes|\psi\rangle_{\beta}\right)$ is the eigenstate $|0\rangle_{\alpha} \otimes|\phi\rangle_{A B \beta}$ of $\left(\sigma_{z}\right)_{\alpha} \otimes \mathbb{1}_{A B \beta}$. Equivalently,

$$
\begin{aligned}
G_{1}\left(|\chi\rangle_{\alpha A B} \otimes|\psi\rangle_{\beta}\right) & =\left(U_{\psi}^{\dagger}|0\rangle_{\alpha}\right) \otimes|\phi\rangle_{A B \beta} \\
& =|\psi\rangle_{\alpha} \otimes|\phi\rangle_{A B \beta} .
\end{aligned}
$$

In other words, the operation $G_{1}$ necessarily transfers Bob's state $|\psi\rangle$ to Alice's qubit $\alpha$. Substituting Eq. (12) into Eq. (7) then shows that $G_{2}$ necessarily transfers $U|\psi\rangle$ back to Bob's qubit $\beta$. From these results and the fact that quantum state teleportation is an optimal 
procedure for local state transfer, we conclude that the optimal procedure for implementing locally a universal $U$-teleportation scheme is by means of bidirectional state teleportation. In this paper we have investigated the potential use of LOCCSE for the remote control of a quantum system. We have determined requirements that must be satisfied by any method that implements this task by LOCCSE means. In particular, we have shown that, if Alice can teleport an arbitrary unitary operation to a qubit in her colleague Bob's laboratory, then she must communicate at least two bits of classical information to him, and they must share at least 2 ebits of entanglement. If the unitary operation is remotely implemented by a classical apparatus, then to effect the teleportation at least 2 classical bits must also be transmitted from Bob to Alice. These resources can be used to perform the teleportation of $U$ using bidirectional state teleportation. Remarkably, no protocol employing a smaller amount of resources is possible.

We believe that this work will stimulate further research into ways in which LOCCSE can be used to control remotely the properties of other quantum system, with potential applications ranging from remotely synchronized time evolutions to distributed quantum computing.

Acknowledgements: The authors thank O. Steuernagel and S. M. Barnett for discussions and D. Jonathan and S. Virmani for critically reading the manuscript. This work has been supported by The Leverhulme Trust, the EQUIP project of the European Union, the Engineering and Physical Sciences Research Council (EPSRC) and DGICYT Project No. PB-98-0191 (Spain).

(*) Permanent Address: Departamento de Física. Universidad de Oviedo. Calvo Sotelo s/n. 33007 Oviedo. Spain. 


\section{REFERENCES}

[1] M.B. Plenio and V. Vedral, Cont. Phys. 39, 431 (1998) and references therein.

[2] J. S. Bell, Speakable and Unspeakable in Quantum Mechanics: Collected Papers on Quantum Philosophy (Cambridge 1987).

[3] C.H. Bennett et al, Phys. Rev. Lett. 70, 1895 (1993).

[4] W.K. Wootters and W.H. Żurek, Nature 299, 802 (1982).

[5] M.A. Nielsen and I.L. Chuang, Phys. Rev. Lett. 79, 321 (1997).

[6] C.H. Bennett and S.J. Wieser, Phys. Rev. Lett. 69, 2881, (1992).

[7] Although subsystems may move from one laboratory to the other for the purposes of classical communication, their locations will be revealed by the measurement, making $\rho_{B \beta \beta^{\prime}}^{i}$ well defined. 


\section{FIGURES}

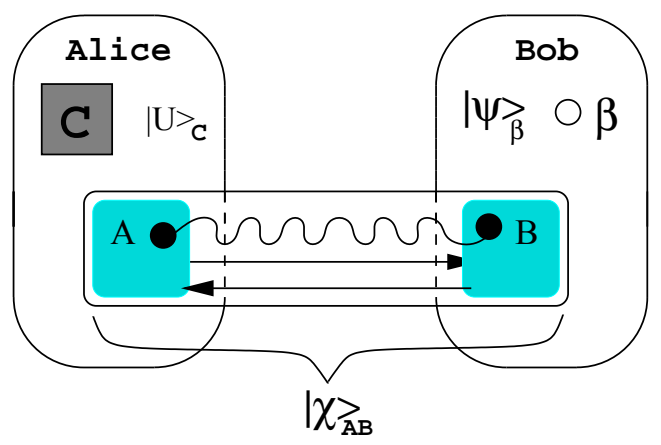

Figure 1. Caption.

Initial setup involved in the teleportation of an arbitrary unitary operation. The control system $\mathrm{C}$ in Alice's laboratory is initially prepared in the state $|U\rangle_{C}$, corresponding to the unitary operation $U$. This operation is to be remotely carried out on Bob's qubit $\beta$, which is initially prepared in an arbitrary pure state $|\psi\rangle_{\beta}$. This will be achieved by local operations in the individual laboratories, involving a collective ancilla initially prepared in the state $|\chi\rangle_{A B}$, supplemented by the exchange of classical communication, represented in the diagram by the arrow lines.

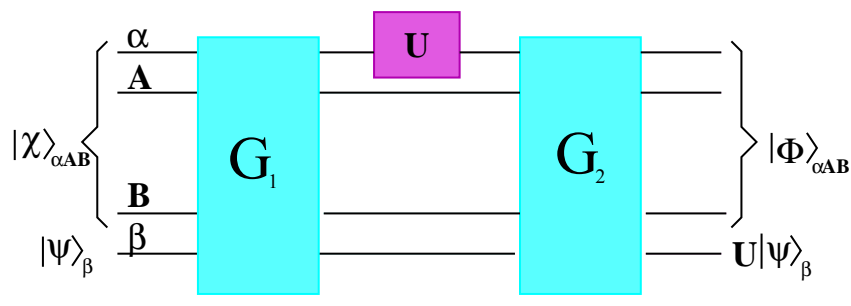

Figure 2. Caption.

Quantum circuit representation of the process of teleporting an arbitrary one-qubit transformation. The two upper wires belong to Alice and the lower ones to Bob. Initially Alice and Bob share some entanglement, represented by the joint state $|\chi\rangle_{\alpha A B}$. Operations $G_{1}$ and $G_{2}$ are modeled in terms of non-local unitary transformations. 